\title{
Throughput Analysis for a Multi-User, Multi-Channel ALOHA Cognitive Radio System
}

\author{
Xiaofan Li, Student Member, IEEE, Hui Liu, Fellow, IEEE, Sumit Roy, Fellow, IEEE, \\ Jianhua Zhang, Member, IEEE, Ping Zhang, Member, IEEE, and Chittabrata Ghosh, Member, IEEE
}

\begin{abstract}
In this paper, we investigate a novel slotted ALOHAbased distributed access cognitive network in which a secondary user (SU) selects a random subset of channels for sensing, detects an idle (unused by licensed users) subset therein, and transmits in any one of those detected idle channels. First, we derive a range for the number of channels to be sensed per SU access. Then, the analytical average system throughput is attained for cases where the number of idle channels is a random variable. Based on that, a relationship between the average system throughput and the number of sensing channels is attained. Subsequently, a joint optimization problem is formulated in order to maximize average system throughput. The analytical results are validated by substantial simulations.
\end{abstract}

Index Terms-Cognitive radio, distributed system, multichannels, throughput analysis, number of sensing channels.

\section{INTRODUCTION}

$\mathbf{T}$ HE surge in demand for high bandwidth applications on mobile devices is driving the current imperative for either additional spectrum allocations and/or more efficient use of existing ones. Cognitive radios (CR) [1] performing dynamic spectrum access seems to be a natural pathway for realizing improved spectrum efficiencies by allowing secondary users on hitherto licensed spectrum. Utilization of licensed band imposes the constraint of sensing channel availability for opportunistic access by unlicensed (secondary) users (SUs), without imposing inadmissible interference to primary users (PUs).

Coexistence of SUs and PUs may be achieved using either a scheduled (centralized) mechanism or a distributed scheme. For a scheduled mechanism, a central control channel is necessary to schedule SUs on spectrum sensing and packet accessing. Among the former, Cordeiro et al. [2] presented

Manuscript received July 29, 2011; revised February 27 and May 19, 2012; accepted August 1, 2012. The associate editor coordinating the review of this paper and approving it for publication was D. Tuninetti.

X. Li, J. Zhang, and P. Zhang are with the Key Laboratory of Universal Wireless Communications for the Ministry of Education, Wireless Technology Innovation Institute, Beijing University of Posts and Telecommunications, Beijing, 100876, China (e-mail: \{xiaofanli, jhzhang, pzhang\}@bupt.edu.cn).

H. Liu is with the Department of Electrical Engineering, Shanghai JiaoTong University, Shanghai, 200240, China (e-mail: huiliu@sjtu.edu.cn).

S. Roy is with the Department of Electrical Engineering, University of Washington, Box 352500 Seattle, WA 98195-2500 (e-mail: sroy@u.washington.edu).

C. Ghosh is with Nokia Research Center, Berkeley, CA-94704 (e-mail: chittabrata.ghosh@nokia.com).

This research is supported by the National Natural Science Foundation of China with No. 61171105, the Program for New Century Excellent Talents in University, NCET-11-0598, the National Basic Research Program of China (973Program) with No. 2009CB320400, and the National Science Foundation Goali Grant NO. 0801997.

Digital Object Identifier 10.1109/TWC.2012.092112.111425 a Cognitive MAC (Media Access Control) protocol over the vacant TV broadcasting spectrum based on instructions from base station and analyzed efficiency improvement of the SU in terms of throughput, delay, and quality of service. Hamdaoui et al. [3] proposed an opportunistic spectrum MAC protocol through periodically listening to a control channel and undertook a simulation based performance evaluation.

For a distributed access, reliable (and fast) spectrum sensing is vital for overall system performance due to the absence of central control channel or scheduling mechanism. In existing serial or random sensing mechanisms - the "classic sensing strategies (CSS)" - each SU serially or randomly searches the channels within the spectrum for an idle channel. The search scheme terminates only when such a channel is identified. The performance of various medium access control (MAC) layer protocols based on such CSS constitute the core of the crosslayer cognitive network performance evaluation literature. In [4] and [5], the authors proposed an opportunistic multichannel MAC protocol and analyzed its throughput using random sensing with negotiation, that required an extra control channel for coordination among SUs. In contrast, Choe et al. [6] and [7] provided analytical results for throughput of a slotted ALOHA-based multi-channel CR system in a proposed Random Channel Selection scheme without any such control coordination channel.

The above were also limited to narrowband systems; more recently, wideband dynamic spectrum access (DSA) systems notably based on orthogonal frequency division multiplexing (OFDM) - have been explored for flexible spectrum pooling as in [8] and [9]. In such scenarios, the SUs should be capable of opportunistically detecting multiple non-contiguous idle OFDM sub-carriers and accessing them. This motivates our present work where we consider a (generic) spectrum pool consisting of a random set of licensed channels that the SUs select for sensing until the first available (idle) channel is detected. However, sensing of multiple idle channels successfully is a more challenging problem, whose performance (as characterized by imperfect decisions with probability of mis-detections and false alarms) in turn affects the MAC in which it is embedded. Novel sensing algorithms are needed that balance the latency (number of samples needed) for detection of primary user occupancy with desired accuracy (given detection error probability).

In this context, Liang et al. [10] studied the performance tradeoff between sensing time and achieved throughput of SUs in point-to-point transmission. While, Quan et al. [11] developed an optimization approach to maximize the aggregate throughput for optimal sensing thresholds for each 
sub-band. To extend the above research, Luo et al. [12] considered joint minimization of the average detection time for finding a spectrum hole as a function of both duration and threshold in the constraint of certain detection and false alarm probabilities. In order to improve sensing performance, an extensive research also by Luo et al. [13] have been pursued based on two stage sensing, which involves coarse resolution detection in the first stage and finite resolution detection in the second stage.

We generalize prior art by allowing each of the SUs to select a (randomly chosen) set of channels, for sensing and determining channel status (idle/occupied). Each SU then undertakes packet transmission in any one of the idle channels from its (detected) idle sub-set. This method is termed "extended sensing strategy (ESS)" which reveals some interesting aspects for further investigation. One is the tradeoff between the number of channels to be sensed and the system throughput. Further, the optimal system throughput by jointly adjusting the packet transmission probability and the number of channels to be sensed by each SU is explored via analytical formulation.

In this paper, we consider the ESS applied to a slotted ALOHA-based multi-channel random access CR system. The major new contributions of this paper are:

- Proposed the Extended Sensing Strategy for multichannel ALOHA access;

- Derive a relationship between the average system throughput and the number of sensing channel, $N_{s}$, find optimal $N_{s}$;

- Consider imperfect sensing and attain the analytical average system throughput;

- Optimize average system throughput jointly with respect to $N_{s}$ and the packet transmission probability;

The rest of the paper is organized as follows. Section II briefly discusses the system model of multi-user multi-channel spectrum access. In Section III, an analytical derivation of the average throughput based on the ESS is provided. Section IV deals with the joint optimization problem with respect to $N_{s}$ and the packet transmission probability. In Section $\mathrm{V}$, the average system throughput under imperfect sensing is derived. Section VI discusses average throughput achieved by comparing the simulation results with those obtained from our theoretical analysis. Section VII concludes the paper.

\section{System Model}

As shown in Fig. 1, we consider a spectrum of $N$ licensed channels, out of which $M$ random channels are idle or unoccupied by the PUs. Further, there are $K$ SUs located in the same local area and synchronized with the PUs that seek to estimate channel status so as to opportunistically access the idle channels without imposing interference to the PUs. Each access frame (AF) has a fixed duration $T_{\mathrm{AF}}$, divided into two parts - the sensing slot, $T_{S}$, and packet transmission slot, $T_{P}$, depicted in Fig. 2.

\section{A. Spectrum Sensing Strategy in one AF}

During the sensing slot, each SU accomplishes sensing one licensed channel in one unit slot $T_{S m}$ within $T_{S}$. Each SU senses $N_{s}$ channels in sequence which are randomly selected

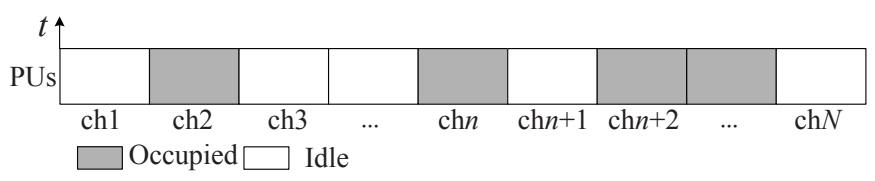

Fig. 1. Channel states of PUs in one slot.

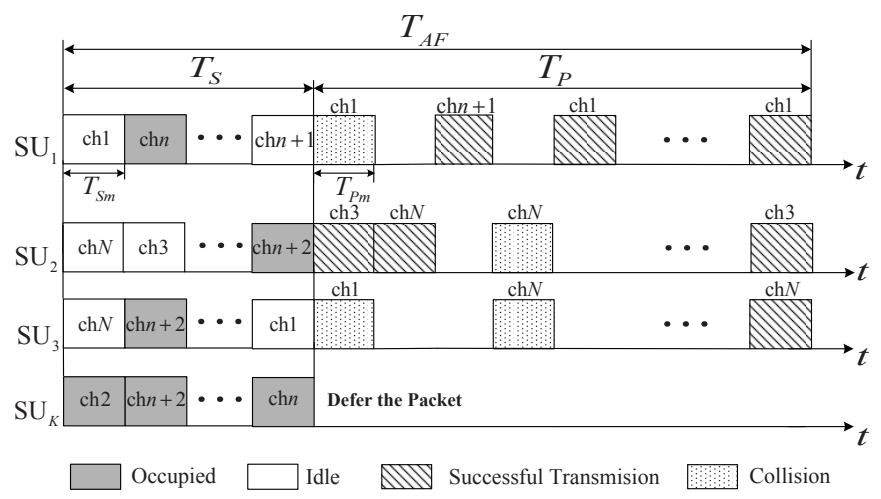

Fig. 2. Access frame for SUs based on the channel states in Fig. 1.

from the $N$ channels, and detects/constructs its idle channel set. We name this as the "extended sensing strategy." The time required for sensing all channels is $T_{S}=T_{S m} N_{s}$; clearly the number of channels determined to be idle by the $k^{\text {th }}$ SU, $M_{s}^{k} \leq \min \left(N_{s}, M\right)$, is a random variable. Due to the absence of any central control channel, one SU will not know which channels are sensed idle by the other SUs. Since the determination of each individual channel status as busy/idle is subject to (occasional) error, determined by the probability of (correct) detection of the presence of PUs' signals $P_{d}$ and probability of false alarm $P_{f}$ (probability of falsely declaring a idle channel as busy), we first assume ideal detection, i.e., $P_{d}=1$ and $P_{f}=0$ for simplicity in this work. The cases involving imperfect sensing, i.e., $P_{d} \neq 1$ and $P_{f} \neq 0$, will be studied in Section $V$.

\section{B. MAC for Packet Transmission in one AF}

For packet transmission, slotted ALOHA access is applied. As shown in Fig. 2, the $k^{\text {th }}$ SU transmits a single packet at the beginning of each unit transmission slot, $T_{P m}$, with probability $p_{\text {tra }}^{k}$ on any one of the $M_{s}^{k}$ idle channels that has been determined to be idle, during the sensing period $T_{S}$. Thus, the number of transmitted packets in one slot, $U$, is a random variable. Take the fairness of packets access in consideration, each SU has the same transmission probability $p_{\text {tra }}\left(p_{\text {tra }} \in[0,1]\right)$. Thus, $E[U]=K p_{\text {tra }}$, due to the independent identical binomial distribution of the packet transmission for each SU.

Since there is no coordination among SUs, each SU accesses one idle channel through a random selection among its own detected idle channel set. Thus, successful packet transmission of the $k^{\text {th }} \mathrm{SU}$ in a time slot only occurs when the following two conditions are satisfied simultaneously:

- $M_{s}^{k} \neq 0$, i.e., at least one idle channel exists within $N_{s}$ sensed channels;

- The $k^{\text {th }}$ SU transmits a packet on an idle channel randomly selected from its $M_{s}^{k}$ idle channel set, that is NOT accessed by any other SU in the same time slot. 
Correspondingly, the following two situations result in failed packet transmission:

- More than one packet is transmitted on the same idle channel in one time slot (e.g., both $\mathrm{SU}_{1}$ and $\mathrm{SU}_{3}$ transmitting packets in ch1 in the first time slot in Fig. 2), defined as a collision.

- Any packet transmission is deferred when no idle channel is detected within the $N_{s}$ sensed channels (e.g., $\mathrm{SU}_{K}$ in Fig. 2).

Assume there is a timer with each SU and the expiry of the timer identifies an unsuccessful transmission. Each lost packet is re-queued and will be retransmitted in a future AF when an idle channel is found. We assume that an SU always has at least one packet in its buffer. Further, the state of PU channels changes sufficiently slowly, such that it can be viewed as constant within a $T_{\mathrm{AF}}$.

Consequently,

$$
T_{\mathrm{AF}}=T_{S}+T_{P}=N_{s} T_{S m}+N_{p} T_{P m}
$$

where we let $T_{P m}=\eta T_{S m}$. For higher throughput, $N_{p}>>$ $N_{s}$ is typical.

\section{Throughput ANALYSIS BASED ON "EXTENDED SENSING STRATEGY"}

In this section, we will analyze the average system throughput based on the "extended sensing strategy". According to the system model description in Section II, we will firstly provide the range of choosing $N_{s}$ channels for sensing. Then the relationship between $N_{s}$ and the average system throughput will be investigated.

\section{A. Choice of $N_{s}$}

We assume that $M(M \leq N)$ among $N$ licensed channels are idle. After selecting (randomly) an $N_{s}$ subset, the $k^{\text {th }}$ SU determines the status of $M_{s}^{k}$ idle channels within the $N_{s}$ channels sensed, where $M_{s}^{k} \leq M$.

Lemma 1. The probability, $p_{\text {sac }}^{(i)}$ of the $i^{\text {th }}$ idle channel being selected for access is given by the following (note that the result is indep. of the index of the idle channel):

$$
p_{s a c}^{(i)}= \begin{cases}\frac{1}{M}\left(1-\frac{\left(N-N_{s}\right)^{[M]}}{N^{M]}}\right), & \text { if } N_{s} \leq N-M, \\ \frac{1}{M}, & \text { if } N_{s} \geq N-M+1,\end{cases}
$$

Clearly, $p_{\text {sac }}^{(i)} \in[1 / N, 1 / M]$.

Proof: Refer to Appendix for the derivation of $p_{\text {sac }}^{(i)}$.

From Lemma 1, we find that a given idle channel selected for a packet access depends on $N_{s}$, but not on $M_{s}^{k}$. Moreover, since $p_{\text {sac }}^{(i)}$ is invariant w.r.t $i$, it implies that $p_{\text {sac }}^{(1)}=p_{\text {sac }}^{(2)}=\ldots=$ $p_{\mathrm{sac}}^{(M)}$ and we may drop the superscript in the sequel. Note that accessing an idle channel does not guarantee a successful packet transmission for reasons described in Section II-B. Based on the result in Lemma 1, the average number of packets in successful transmission can be derived as followings, when there are $U$ packets transmitted from all the SUs in a slot.
When all users transmit packets with $p_{\text {tra }}=1$, i.e. they always have a packet to send in each slot, $U=K$.

Theorem 1. When $U$ packets are sent to access the $M$ idle channels, the average number of successfully transmitted packets is given by

$$
S_{\text {suc }}(U)=M U p_{s a c}\left(1-p_{s a c}\right)^{U-1} .
$$

Additionally, $S_{\text {suc }}(U)$ is constant if $N_{s}>N-M+1$.

Proof: From Appendix, $X_{i}=\sum_{j=1}^{U} X_{i j}$ is the number of packets accessing the $i^{\text {th }}$ idle channel in one time slot. We introduce a new binary random variable $Z_{i}$, that equals 1 if the $i^{t h}$ idle channel is accessed by only one packet, otherwise, $Z_{i}=0$, if no packet or more than one packet accesses the $i^{\text {th }}$ idle channel. That means $p\left(Z_{i}=1\right)=p\left(X_{i}=1\right)$.

Since successful transmissions only occur if an idle channel is accessed by a single packet, the average number of successful transmissions in a slot $S_{\text {suc }}(U)$ is expressed as

$$
\begin{aligned}
S_{\text {suc }}(U) & =E[Z \mid U]=E\left[\sum_{i=1}^{M} Z_{i} \mid U\right] \\
& =\sum_{i=1}^{M} E\left[Z_{i} \mid U\right]=\sum_{i=1}^{M} p\left(Z_{i}=1 \mid U\right),
\end{aligned}
$$

where $Z=\sum_{i=1}^{M} Z_{i}$ is the number of idle channels with only one packet access. Since $p\left(X_{1}=1 \mid U\right)=p\left(X_{2}=1 \mid U\right)=$ $\ldots=p\left(X_{M}=1 \mid U\right)$ and each $X_{i j}$ represents a independent Bernoulli trial, we can reformulate (4) as,

$$
\begin{aligned}
S_{s u c}(U) & =\sum_{i=1}^{M} p\left(X_{i}=1 \mid U\right)=M p\left(X_{i}=1 \mid U\right) \\
& =M p\left(\sum_{j=1}^{U} X_{i j}=1 \mid U\right)=M\left(\begin{array}{l}
U \\
1
\end{array}\right) p_{\mathrm{sac}}\left(1-p_{\mathrm{sac}}\right)^{U-1}
\end{aligned}
$$

yielding (3).

From (3), we deduce that $N_{s}$ is independent of $S_{s u c}$ when $N_{s}>N-M+1$. It implies that the number of successful packet transmissions will not always increase with increasing values of $N_{s}$, i.e. it is not necessary to choose more than $N-M+1$ channels for sensing. We will verify this fact from the simulation results discussed in Section VI.

\section{B. Average System Throughput}

Define the average system throughput as the average number of packets in successful transmission per slot. Following Theorem 1, we first derive the expression of the average system throughput, when each SU transmits the packet with $p_{\text {tra }}<1$ and $U$ is a random variable. Then the average system throughput under a random $\mathbf{M}$ is obtained.

Theorem 2. The average system throughput conditioned on $M$ is

$$
S_{s y s}\left(N_{s}, p_{\text {tra }}\right)=\frac{\eta N_{p}}{N_{s}+\eta N_{p}} M K p_{\text {sac }} p_{\text {tra }}\left(1-p_{\text {tra }} p_{\text {sac }}\right)^{K-1} .
$$




$$
\begin{aligned}
S_{\text {sys }}\left(N_{s}, p_{\text {tra }}\right) & =E\left[\frac{N_{p} T_{P m} S_{\text {suc }}(U)}{N_{s} T_{S m}+N_{p} T_{P m}}\right] \\
& =\frac{N_{p} T_{P m}}{N_{s} T_{S m}+N_{p} T_{P m}} E\left[S_{\text {suc }}(U)\right] \\
& =\frac{\eta N_{p}}{N_{s}+\eta N_{p}} E\left[S_{s u c}(U)\right] .
\end{aligned}
$$

We define $S_{\text {ave }}=E\left[S_{\text {suc }}(U)\right]$ as the average number of the packets in successful transmission. Using Theorem 1, $S_{\text {ave }}$ for a given $M$ can be written as,

$$
\begin{aligned}
S_{\mathrm{ave}} & =E\left[M U p_{\mathrm{sac}}\left(1-p_{\mathrm{sac}}\right)^{(U-1)}\right] \\
& =M p_{\mathrm{sac}} E\left[U\left(1-p_{\mathrm{sac}}\right)^{(U-1)}\right] \\
& =M p_{\mathrm{sac}} \sum_{U=1}^{K} U\left(1-p_{\mathrm{sac}}\right)^{U-1} p_{\mathrm{num}}(U),
\end{aligned}
$$

where $p_{\text {num }}(U)$ is the probability that there are $U$ packets transmitted from $K$ SUs. Since the packet transmission of each $\mathrm{SU}$ is the independent Binomial distribution, $p_{\text {num }}(U)$ can be obtained as

$$
p_{\text {num }}(U)=\left(\begin{array}{c}
K \\
U
\end{array}\right)\left(p_{\text {tra }}\right)^{U}\left(1-p_{\text {tra }}\right)^{K-U} .
$$

Substituting (9) into (8) simplifies to

$$
\begin{aligned}
S_{\text {ave }} & =M p_{\text {sac }} K p_{\text {tra }} \sum_{U=1}^{K}\left(1-p_{\text {sac }}\right)^{U-1}\left(\begin{array}{l}
K-1 \\
U-1
\end{array}\right)\left(p_{\text {tra }}\right)^{U-1}\left(1-p_{\text {tra }}\right)^{K-U} \\
& =M K p_{\text {sac }} p_{\text {tra }}\left(p_{\text {tra }}\left(1-p_{\text {sac }}\right)+1-p_{\text {tra }}\right)^{K-1} \\
& =M K p_{\text {sac }} p_{\text {tra }}\left(1-p_{\text {tra }} p_{\text {sac }}\right)^{K-1} .
\end{aligned}
$$

yielding (6).

Note that (6) is dependent on $N, M$ and $K$ whereby each SU senses the idle channels and transmits the packet with a fixed $\left(N_{s}, p_{\text {tra }}\right)$. Indeed, in a distributed system, the number of idling channels and the number of SUs are generally not known to the SUs. Hence, these parameters need to be estimated at the SU side without a control channel. In the practical system, $N$ is generally known by each SU due to awareness of the range of the spectrum allowed for SUs access. Thus, the remaining question is how to obtain $K$ and $M$ based on the outputs of the initial access period (IAP). It is noted that each SU is aware of the packet in successful (failed) transmission and can count the total number of the idle channels detected during all the sensing slots in IAP. Thus, $K$ and $M$ can be estimated based on individual SU's mean throughput value [14]. In this paper, we assume each SUs has the perfect knowledge about the system parameters $K$ and $M$.

In a dynamic environment, the PU is silent on a channel with probability $q$ and active with probability $1-q$. That implies that the number of idle channels $\mathbf{M}$ is a random variable. To avoid interference from SU to the PUs, we assume that the PU's state is fixed within the access period. Accordingly we have the following result.

Corollary 1. The average system throughput under a random $\boldsymbol{M}, S_{r m}\left(N_{s}, p_{\text {tra }}\right)$, is

$$
\begin{aligned}
& S_{r m}\left(N_{s}, p_{\text {tra }}\right)=\frac{\eta N_{p}}{N_{s}+\eta N_{p}} K p_{\text {tra }} \\
& \cdot \sum_{m=1}^{N} m p_{\text {sac }}(m)\left(1-p_{\text {tra }} p_{\text {sac }}(m)\right)^{K-1}\left(\begin{array}{l}
N \\
m
\end{array}\right) q^{m}(1-q)^{N-m} .
\end{aligned}
$$

Proof: Since the PU channels are iid, the probability of $\mathbf{M}=m$ idle channels among $N$ PU channels is given by

$$
P_{\text {numidl }}(\mathbf{M}=m)=\left(\begin{array}{c}
N \\
m
\end{array}\right) q^{m}(1-q)^{N-m}
$$

According to (6), the average system throughput under a random $\mathbf{M}$ can be derived as

$$
\begin{aligned}
S_{r m}\left(N_{s}, p_{\text {tra }}\right)=E\left[S_{s y s}\left(N_{s}, p_{\text {tra }} \mid \mathbf{M}\right)\right] \\
\quad=\sum_{m=1}^{N} \frac{\eta N_{p} m K p_{\text {tra }} p_{\text {sac }}(m)}{N_{s}+\eta N_{p}}\left(1-p_{\text {tra }} p_{\text {sac }}(m)\right)^{K-1} P_{\text {numidl }}(m) \\
\quad=\frac{\eta N_{p} K p_{\text {tra }}}{N_{s}+\eta N_{p}} \sum_{m=1}^{N} m p_{\text {sac }}(m)\left(1-p_{\text {tra }} p_{\text {sac }}(m)\right)^{K-1} P_{\text {numidl }}(m),
\end{aligned}
$$

yielding (11).

In several scenarios, $M$ is slowly varying relative to the $\mathrm{SU}$ access frame duration. Hence for throughput maximization, we first fix the number of idle channels so that other system parameters (e.g., the SU's access rate) can be jointly optimized. Subsequently, we average over the distribution of a random $\mathbf{M}$ to explore the impact of varying $M$ over a sufficiently long period.

\section{Relationship between $N_{s}$ and Average System Throughput}

From (6), it is observed that the average system throughput will be affected by both $N_{s}$ and $p_{\text {tra }}$. Since $N_{s}$ is a discrete and finite variable, we can in principle obtain the optimal $N_{s}$ via exhaustive search in (6) and selecting the $N_{s}$ corresponding to the maximal $S_{\text {ave }}$. However, this is not effective for $N_{s}$ increasing or variable $p_{\text {tra }}$.

Proposition 1. For the maximal average system throughput, $N_{s}$ and $S_{\text {sys }}$ for a given $p_{\text {tra }}$ are related via

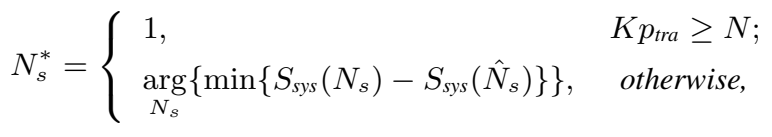

where $\hat{N}_{s}$ satisfies $\left.\frac{\partial S_{s y s}}{\partial N_{s}}\right|_{\hat{N}_{s}}=0$ and $\bar{N}_{s} \in[0, N-M+1]$.

Proof: We relax $N_{s}$ as $\bar{N}_{s} \in[0, N-M+1]$ and differentiate $S_{\text {sys }}$ in (6) with respect to $\bar{N}_{s}$.

i) When $K p_{\text {tra }}>N$, which means $\frac{1}{K p_{\text {tra }}}<\frac{1}{N} \leq p_{\text {sac }}$, then $1-K p_{\text {tra }} p_{\text {sac }}<0$ and $\frac{\partial S_{\text {sys }}}{\partial N_{s}}<0$. That indicates $S_{\text {sys }}$ decreases with $p_{s a c}$. Further, $p_{s a c}$ is an increasing function of $N_{s}$ as proved in Appendix. Therefore, $S_{\text {ave }}$ achieves the maximal value at $N_{s}^{*}=1$.

ii) $K p_{\text {tra }}<N$, i.e., $\frac{1}{N} \leq \frac{1}{K p_{\text {tra }}}$, then $\frac{\partial S_{\text {sys }}}{\partial N_{s}}>0$ at $\bar{N}_{s}=0$ and $\frac{\partial S_{\mathrm{sys}}}{\partial N_{s}}<0$ at $\bar{N}_{s}=N-M+1$. Due to the continuity of 
$\frac{\partial S_{\text {sys }}}{\partial N_{s}}$ on $\bar{N}_{s} \in[0, N-M+1]$, there exists a $\hat{N}_{s}$ which satisfies $\left.\frac{\partial S_{\text {sys }}}{\partial \bar{N}_{s}}\right|_{\hat{N}_{s}}=0$. Further, once $\left.\frac{\partial S_{\text {sys }}}{\partial \bar{N}_{s}}\right|_{\hat{N}_{s}}=0,\left.\frac{\partial^{2} S_{\text {sys }}}{\partial \bar{N}_{s}^{2}}\right|_{\hat{N}_{s}}<0(K>$ $2)$, which means $S_{\text {sys }}\left(\hat{N}_{s}\right)$ is the only maximal point on the domain. Thus, $\left.N_{s}^{*}=\underset{N_{s}}{\arg \{\min }\left\{S_{\text {sys }}\left(N_{s}\right)-S_{\text {sys }}\left(\hat{N}_{s}\right)\right\}\right\}$.

From Proposition 1 , if $K p_{\text {tra }} \geq N$, each SU can directly set $N_{s}^{*}=1$ without extra searching. While, if $K p_{\text {tra }}<N$, each SU can obtain $N_{s}^{*}$ by calculating $\hat{N}_{s}$. When it is not convenient to compute $N_{s}$ directly, we apply the first order and second order partial derivatives for the optimal $S_{\text {sys }}$ as in the proof of Proposition 1, i.e., $\left.\frac{\partial^{2} S_{\mathrm{sys}}}{\partial N_{s}^{2}}\right|_{\hat{N}_{s}}<0$, once $\left.\frac{\partial S_{\mathrm{sys}}}{\partial N_{s}}\right|_{\hat{N}_{s}}=0$. This implies that when the first order derivative of $S_{\text {sys }}$ at two adjacent $N_{s}$ values are different in sign, $N_{s}^{*}$ corresponds to the larger $S_{\text {sys. }}$. Choice of a proper initial value for $N_{s}$ will result in more effective searching in such cases, but is beyond the scope of our paper.

Moreover, as $E[U]=K p_{\text {tra }}$, that means when the average number of transmitted packets is larger than $N$, each SU does not need to sense the channels for more than one $T_{S m}$. That is because it will introduce more collisions and thereby reduce the system throughput, although each SU incurs more time and resources on sensing the channels. For $E[U]=K p_{\text {tra }}<N$, it is worth investing more time on sensing the idle channel for each SU and the optimal $N_{s}$ can be decided according to Proposition 1. With this understanding, let us focus on the system throughput optimization with respect to both $N_{s}$ and $p_{\text {tra }}$ in the next section.

\section{JOINT OPTIMIZATION ON SYSTEM THROUGHPUT MAXIMIZATION THROUGH $\left(N_{s}, p_{\text {tra }}\right)$}

In this section, we focus on the throughput maximization jointly w.r.t. $N_{s}$ and $p_{\text {tra. }}$ According to (6), the problem can be presented as

$$
\begin{gathered}
\underset{\left(N_{s}, p_{\text {tra }}\right)}{\arg } \max S_{\text {sys }}=\frac{\eta N_{p}}{N_{s}+\eta N_{p}} M K p_{\text {sac }} p_{\text {tra }}\left(1-p_{\text {tra }} p_{\text {sac }}\right)^{K-1} \\
\text { s.t. } 0 \leq p_{\text {tra }} \leq 1 \\
\quad N_{s} \in\{1,2, \ldots, N-M+1\} .
\end{gathered}
$$

Since the above is neither convex nor concave on the domain of $N_{s}$ or $p_{\text {tra }}$, that implies the Karush-Kuhn-Tucker conditions will only provide locally optimal solution. Thus, in the following, we analyze the optimal $p_{\text {tra }}$ according to the relationship between $N_{s}^{*}$ and throughput for a given $p_{\text {tra }}$ which we have obtained in Proposition 1, and finally obtain the optimal pair $\left(N_{s}, p_{\text {tra }}\right)$.

Proposition 2. For a given $N_{s}$, the optimal $p_{\text {tra }}$ for maximizing the system average throughput in (15a) is given by,

$$
p_{\text {tra }}^{*}=\left\{\begin{aligned}
\frac{1}{K p_{\text {sac }}}, & p_{\text {sac }}>1-1 / K \\
1, & p_{s a c} \leq 1-1 / K .
\end{aligned}\right.
$$

Proof: Differentiate $S_{\text {sys }}$ in (15a) with respect to $p_{\text {tra }}$. When $\left.\frac{\partial S_{\text {sys }}}{\partial p_{\text {tra }}}\right|_{\tilde{p}_{\text {tra }}}=0, \tilde{p}_{\text {tra }}=\frac{1}{K p_{\text {sac }}}$ and $\left.\frac{\partial^{2} S_{\text {sys }}}{\partial p_{\text {tra }}{ }^{2}}\right|_{\tilde{p}_{\text {tra }}}<0$. Thus, $S_{\text {sys }}\left(\tilde{p}_{\text {tra }}\right)$ is the only maximum value on the given $N_{s}$. Further, when $p_{\text {tra }}<\tilde{p}_{\text {tra }},\left.\frac{\partial S_{\text {sys }}}{\partial p_{\text {tra }}}\right|_{<\tilde{p}_{\text {tra }}}>0$, implying that (15a) is an increasing function in this rang. Thus, the optimal $p_{\text {tra }}$ is attained at $p_{\text {tra }}^{*}=\min \left\{\frac{1}{K p_{\text {sac }}}, 1\right\}$.

In terms of Proposition 2, for $p_{\mathrm{sac}} \leq 1 / K$, each SU should always transmit the packet in each $T_{S m}$ slot. For $p_{\mathrm{sac}}>1 / K$, each SU will reduce the transmission probability to $\frac{1}{K p_{\text {sac }}}$ to reduce the chance of collisions. Moreover, $N, M$, and $K$ also affects the optimal packet transmission probability in the range $p_{\text {sac }} \in[1 / N, 1 / M]$. Take all this in consideration, we develop the optimal $\left(N_{s}, p_{\text {tra }}\right)$ as follows.

Theorem 3. The maximal average system throughput can be attained at the optimal values of $\left(N_{s}, p_{\text {tra }}\right)$ given by

$$
S_{\max }=\left\{\begin{array}{lr}
S_{s y s}\left(N_{s 1}^{*}, p_{\text {tra } 1}^{*}\right), & K \leq M \\
S_{s y s}\left(N_{s 2}^{*}, p_{\text {tra } 2}^{*}\right), & K \geq N \\
\max \left(S_{s y s}\left(N_{s 1}^{*}, p_{\text {tra } 1}^{*}\right), S_{s y s}\left(N_{s 2}^{*}, p_{\text {tra } 2}^{*}\right)\right), \text { others }
\end{array}\right.
$$

where $\left(N_{s 1}^{*}, p_{\text {tra1 }}^{*}\right)=\left(\underset{N_{s}}{\arg \min _{p_{\text {tra }}=1}}\left\{S_{\text {sys }}\left(N_{s}\right)-S_{\text {sys }}\left(\hat{N}_{s}\right)\right\}, 1\right)$ and $\left(N_{s 2}^{*}, p_{\text {tra } 2}^{*}\right)=(1, N / K)$.

Proof: From Proposition 2, we see that the optimal $\left(N_{s}^{*}, P_{\text {tra }}^{*}\right)$ depends on the relationship between the range $p_{\text {sac }} \in[1 / N, 1 / M]$ and $K$.

a) If $K \leq M$, then there is $p_{\mathrm{sac}} \leq 1 / M \leq 1 / K$. Thus, $p_{\text {tra }}=1$ at any $N_{s}$ value to maximize the average system throughput based on the Proposition 2. Substituting $p_{\text {tra }}=1$ into (15a), the maximal system throughput can be attained at

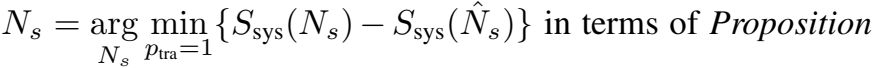
1. Hence, the optimal point is

$$
\left(N_{s 1}^{*}, p_{\text {tra1 }}^{*}\right)=\left(\underset{N_{s}}{\arg \min _{\text {tra }}=1}\left\{S_{\text {sys }}\left(N_{s}\right)-S_{\text {sys }}\left(\hat{N}_{s}\right)\right\}, 1\right) .
$$

b) If $K \geq N$, then $p_{\text {sac }}>1 / N>1 / K$. From Proposition 2, $S_{\text {sys }}$ attains the maximal point at $p_{\text {tra }}=\frac{1}{K p_{\text {sac }}}$ on a given $N_{s}$. That implies for each feasible $N_{s}$ that satisfies $p_{\text {tra }}=\frac{1}{K p_{\mathrm{sac}}}$, the system can achieve the maximal average throughput by adjusting the probability of packet transmission according to (15a). Substituting $p_{\text {tra }}=\frac{1}{K p_{\mathrm{sac}}}$ into (15a), the maximal system throughput will be at $N_{s}=1$. Hence, $S_{\text {sys }}$ attains the maximal point given by,

$$
\left(N_{s 2}^{*}, p_{\text {tra2 }}^{*}\right)=\left(1, \frac{N}{K}\right)
$$

c) For $M<K<N$, we exam $p_{\text {sac }}$ for two cases. For the case when $1 / N<p_{\text {sac }} \leq 1 / K$, the local maximal point is attained at $S_{\text {sys }}\left(N_{s 1}^{*}, p_{\text {tra1 } 1}^{*}\right)$. For the other case $1 / K<p_{\text {sac }}<$ $1 / M$, the system throughput achieves a local maximal point represented as $S_{\text {sys }}\left(N_{s 2}^{*}, p_{\text {tra2 }}^{*}\right)$. Thus, the maximal point will be the maximum value of the above two situations.

Based on Theorem 3, the optimal parameters $\left(N_{s}, p_{\text {tra }}\right)$ can be decided according to the relationship among $K, M$, and $N$. On the other hand, the SUs are able to adjust its own parameters $\left(N_{s}, p_{\text {tra }}\right)$ in order to optimize the system throughput even without the central control channel. We will also verify this conclusion in Theorem 3 through the simulation results. 


\section{System Throughrut ANALYSIS UNDER IMPERFECT SPECTRUM SENSING}

To consider the imperfect sensing [10], [12] and [15], denote $\tau$ as the spectrum sensing time, $f_{s}$ the sampling frequency, and $\gamma$ the received signal-to-noise ratio (SNR) from PU to SUs. The false alarm probability $P_{f}$ can be calculated ([10]) by

$$
P_{f}(\tau)=\mathcal{Q}\left(\sqrt{2 \gamma+1} \mathcal{Q}^{-1}\left(P_{d}\right)+\sqrt{\tau f_{s}} \gamma\right)
$$

where $\mathcal{Q}(\cdot)$ is the complementary function of a standard Gaussian variable and $P_{d}$ is the predefined detection probability.

Assume the detection result indicator of the $n$th channels is $D_{n}(n \in\{1,2, \ldots, N\})$. It is noted that $D_{n}$ indicates the detection results of the idle channels when $n \in\{1,2, \ldots, M\}$, and the occupied channels when $n \in\{M+1, M+2, \ldots, N\}$. If $D_{n}=1$, the $n$th channel is detected as idle channel, otherwise, $D_{n}=0$. The probability of one idle channel detected with no false alarm is $1-P_{f}$ and the probability for an occupied channels detected as an idle channel is $1-P_{d}$. In other words,

$$
\operatorname{Pr}\left(D_{n}=1\right)=\left\{\begin{array}{l}
V_{0}=1-P_{f}, \text { if } n \text {th channel is idle; } \\
V_{1}=1-P_{d}, \text { if } n \text {th channel is occupied }
\end{array}\right.
$$

And the probability of the $i$ th idle channel is detected with no false alarm by one $\mathrm{SU}$ is $\operatorname{Pr}\left(D_{i}=1\right)=V_{0}$.

To obtain the analytical result on the average system throughput under imperfect sensing, we start by rederiving the probability of the $i$ th idle channel selected for packet accessing

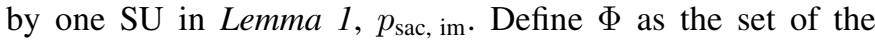
detected channels by one SU. First, the probability that the $i$ th channel is scanned and included in the $M_{s}$ idle channels out of the $N_{s}$ sensed channels is expressed as,

$$
\begin{aligned}
& \operatorname{Pr}\left(\text { the } i \text { th idle channel is scanned } \mid M_{s}\right) \\
& =\left(\begin{array}{c}
M-1 \\
M_{s}-1
\end{array}\right)\left(\begin{array}{c}
N-M \\
N_{s}-M_{s}
\end{array}\right) /\left(\begin{array}{c}
N \\
N_{s}
\end{array}\right) .
\end{aligned}
$$

Assume $M_{D}\left(M_{D} \in\left[1, N_{s}\right]\right)$ channels are detected as idle channels within the $N_{s}$ sensed channels. Out of the $M_{D}$ detected idle channels, the number of the idle channels detected correctly is $m_{\mathrm{ID}}\left(m_{\mathrm{ID}} \in\left[\max \left\{1, M_{s}-\left(N_{s}-\right.\right.\right.\right.$ $\left.\left.\left.\left.M_{D}\right)\right\}, \min \left\{M_{s}, M_{D}\right\}\right]\right)$ and the number of the occupied channels detected as idle status is $m_{\mathrm{OD}}\left(m_{\mathrm{OD}}=M_{D}-m_{\mathrm{ID}}\right)$. Thus, conditioning on $M_{D}$ and $M_{s}$, the probability of the $i$ th idle channel being detected as idling is,

$$
\begin{aligned}
& \operatorname{Pr}\left(D_{i}=1 \mid M_{s}, M_{D}\right) \\
& =\operatorname{Pr}\left(D_{i}=1\right) \cdot \operatorname{Pr}\left(\sum_{n \neq i, n \in \Phi} D_{n}=M_{D}-1 \mid M_{s}\right) \\
& =V_{0} \cdot \sum_{m_{\mathrm{ID}}=\max \left\{1, M_{D}-\left(N_{s}-M_{s}\right)\right\}}^{\min \left\{M_{D}, M_{s}\right\}}\left(\begin{array}{c}
M_{s}-1 \\
m_{\mathrm{ID}}-1
\end{array}\right)\left(V_{0}\right)^{m_{\mathrm{ID}}-1} \\
& \cdot\left(1-V_{0}\right)^{M_{s}-m_{\mathrm{ID}}}\left(\begin{array}{c}
N_{s}-M_{s} \\
m_{\mathrm{OD}}
\end{array}\right)\left(V_{1}\right)^{m_{\mathrm{OD}}}\left(1-V_{1}\right)^{N_{s}-M_{s}-m_{\mathrm{OD}}} .
\end{aligned}
$$

Substituting $m_{\mathrm{OD}}=M_{D}-m_{\mathrm{ID}}$ into (23),

$$
\begin{aligned}
& \operatorname{Pr}\left(D_{i}=1 \mid M_{s}, M_{D}\right) \\
& =\sum_{m_{\mathrm{ID}}=\max \left\{1, M_{D}-N_{s}+M_{s}\right\}}^{\min \left\{M_{D}, M_{s}\right\}}\left(\begin{array}{c}
M_{s}-1 \\
m_{\mathrm{ID}}-1
\end{array}\right) V_{0}^{M_{\mathrm{ID}}}\left(1-V_{0}\right)^{M_{s}-m_{\mathrm{ID}}} \\
& \cdot\left(\begin{array}{l}
N_{s}-M_{s} \\
M_{D}-m_{\mathrm{ID}}
\end{array}\right)\left(V_{1}\right)^{M_{D}-m_{\mathrm{ID}}}\left(1-V_{1}\right)^{N_{s}-M_{s}-\left(M_{D}-m_{\mathrm{ID}}\right)} .
\end{aligned}
$$

With the $M_{D}$ detected idle channels (including the $i$ th idle channel), the probability of a packet accessing the $i$ th idle channel is $1 / M_{D}$. Then, with $M_{s}$ scanned channels and $M_{D}$ detected channels, the probability of the $i$ th idle channel is selected for packet access is obtained as following,

$$
\begin{aligned}
p_{\text {sac, im } \mid\left(M_{s}, M_{D}\right)}^{i} & =\operatorname{Pr}\left(\text { the } i \text { th idle channel is scanned } \mid M_{s}\right) \\
& \cdot \frac{1}{M_{D}} \cdot \operatorname{Pr}\left(D_{i}=1 \mid M_{s}, M_{D}\right) .
\end{aligned}
$$

Hence, the probability of the packet from one SU accessing a specific idle channels is

$$
p_{\mathrm{sac}, \mathrm{im}}^{i}=\sum_{M_{s}=\max \left\{1, N_{s}-(N-M)\right\}}^{\min \left\{N_{s}, M\right\}} \sum_{M_{D}=1}^{N_{s}} p_{\mathrm{sac}, \mathrm{im} \mid\left(M_{s}, M_{D}\right)}^{i} .
$$

It is observed that $p_{\mathrm{sac}, \text { im }}^{i}$ is invariant w.r.t $i$. We may drop the superscript for simplicity: $p_{\mathrm{sac}, \mathrm{im}}$. Given $p_{\mathrm{sac}, \mathrm{im}}$, the average system throughput under imperfect spectrum sensing $S_{\text {sys, im }}$ can be presented as following, according to (6),

$$
S_{\text {sys, im }}=\frac{\eta N_{p}}{N_{s}+\eta N_{p}} M K p_{\text {tra }} p_{\text {sac, im }}\left(1-p_{\text {sac, im }}\right)^{K p_{\text {tra }}-1}
$$

\section{Simulation Results}

In this section, we present the results of the average system throughput via Monte-Carlo simulation. In our simulation, We have considered $N=10$ licensed channels. The sampling frequency $f_{s}$ is set as $6 \mathrm{MHz}$. The overall detection probability for SU is always kept at $P_{d}=0.9$ under imperfect spectrum sensing. The length of one sensing unit, $T_{S m}$, is set at 2 $\mathrm{ms}$. The duration of one unit transmission time is set to be $2 T_{S m}$, i.e., $\eta=2$. Since the actual spectrum sensing time $\tau \in\left(0, T_{S m}\right]$ may vary, we only consider the minimum $P_{f}$ attained at $\tau=T_{S m}$ in our simulations. And the number of packet transmission slots is set as $N_{p}=30$. The $M$ idle channels are randomly located within the $N$ channels. Within an access frame duration $T_{\mathrm{AF}}$, each SU randomly selects $N_{s}$ channels for sensing in $T_{S}$ and transmits the packet on any one of the idle channels identified as idle among the $N_{s}$ channels selected.

The average throughput is obtained by counting the number of the idle channels which have only one packet access in each time slot within $N_{p}$ packet transmissions slots. We apply the free space propagation model with the path-loss exponent $\beta=2$ in our simulations as in [16]. The SNR at SU receiver can be expressed as,

$$
\gamma=P_{\mathrm{S}, \mathrm{PU}} \cdot D_{s u}{ }^{-\beta} / N_{0},
$$




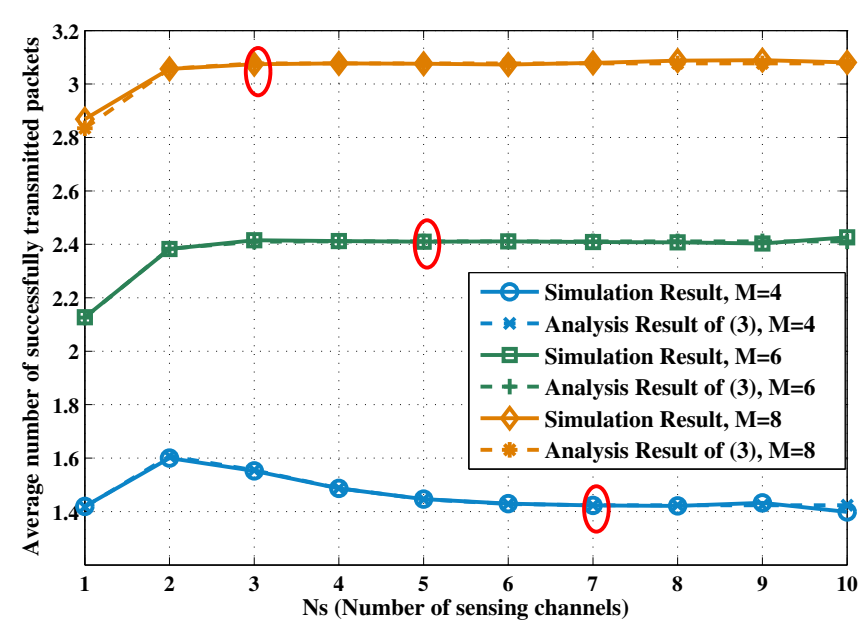

Fig. 3. Average number of successfully transmitted packets in $p_{\text {tra }}=1$ and $K=6$.

where $P_{\mathrm{S}, \mathrm{PU}}$ is the transmission power from the PU and $D_{s u}$ is the distance between the SUs and PU. $N_{0}$ is the average power of noise, which is assumed to be the same for each SU. Moreover, we assume the worst $\gamma$ at an SU receiver is $-20 \mathrm{~dB}$.

Fig. 3 and Fig. 4 present the average number of packets in successful transmissions when each SU transmits the packet with $p_{\text {tra }}=1$. First, we see that the analytical results in (3) fit well with the simulation results in Fig. 3 and Fig. 4, respectively. The average number of successful transmitted packets is constant when $N_{s}>N-M$ as circled points in Fig. 3 and Fig. 4, which means it is independent on $N_{s}$ as specified in Lemma 1. It is intuitive that the average number of successful transmitted packets increases with $M$. Further, when $K<M$, the average number of packets in successful transmission increases with $N_{s}$ until $N_{s}=N-M+1$. Conversely, when $K>N$, the average number of successful transmitted packets declines as $N_{s}$ increases. For $M \leq K \leq N$, it is noted that the number of successful transmitted packets first increases with $N_{s}$, then decreases after the maximum point. This occurs because increasing $N_{s}$ ultimately brings more collisions and reduces successful transmissions.

In Fig. 5 and Fig. 6, the average system throughput are depicted when the probability of packet transmission is given. The analytical results in (6) and the simulation results almost coincide with each other. It can been observed that if $K p_{\text {tra }}>N$, e.g. $K=15$, the throughput is decreasing with $N_{s}$ raising, thus the optimal $N_{s}^{*}=1$. For $K p_{\text {tra }} \leq N$, the throughput achieves the maximal point according to Proposition 1. Further, the throughput in $K=3$ and $K=6$ are larger than that in $K=15$ as $N_{s}$ growing shown in Fig. 6, since more collisions result.

Fig. 7 depicts the maximal throughput of the system with increasing $K$ and the optimal pair $\left(N_{s}, p_{\text {tra }}\right)$ corresponding to the maximal throughput are enumerated in Table I. From Fig. 7 , the analytical results of the system throughput are matching closely with that of the simulation results. Moreover, the throughput improves with increasing number of idle channels in the spectrum. Moreover, there exists an optimal number of

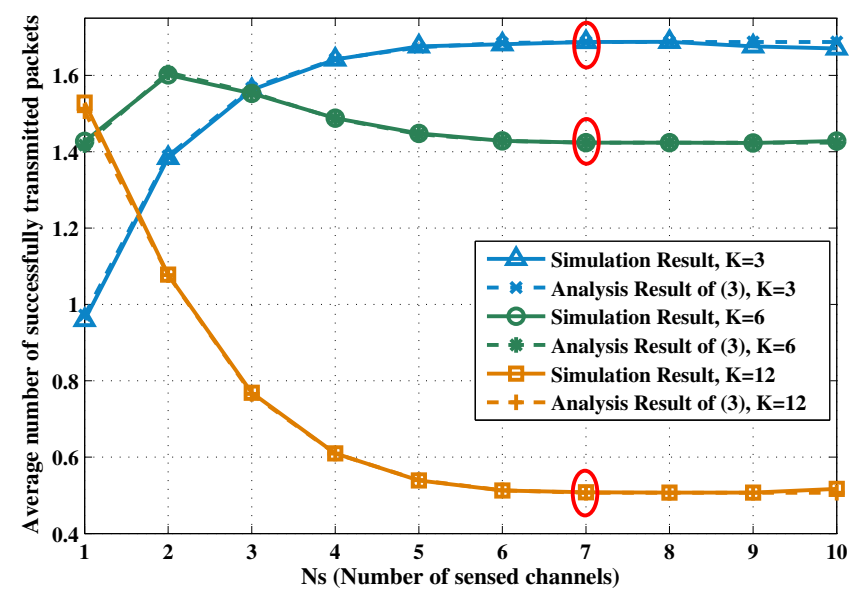

Fig. 4. Average number of successfully transmitted packets in $p_{\text {tra }}=1$ and $M=4$.

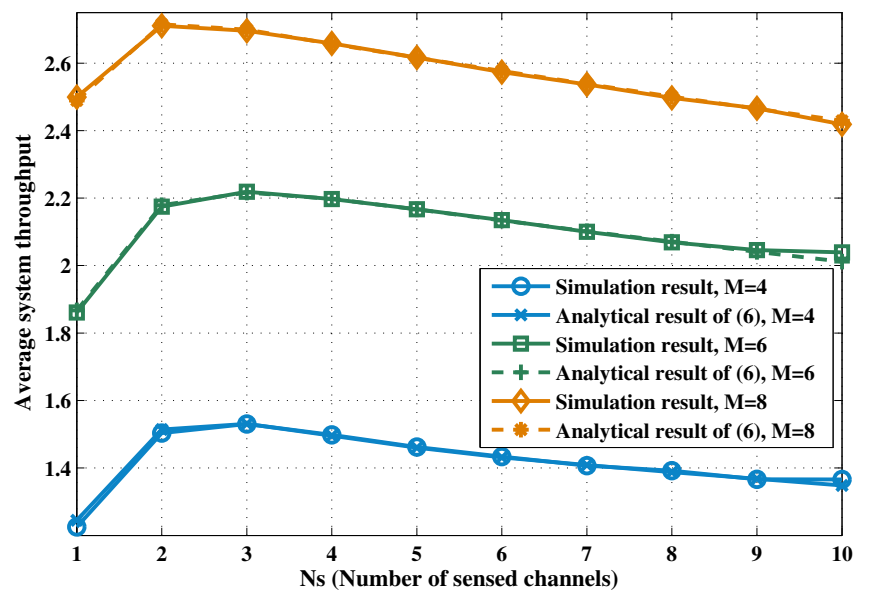

Fig. 5. Average system throughput when $K=6$ and $p_{\text {tra }}=0.8$.

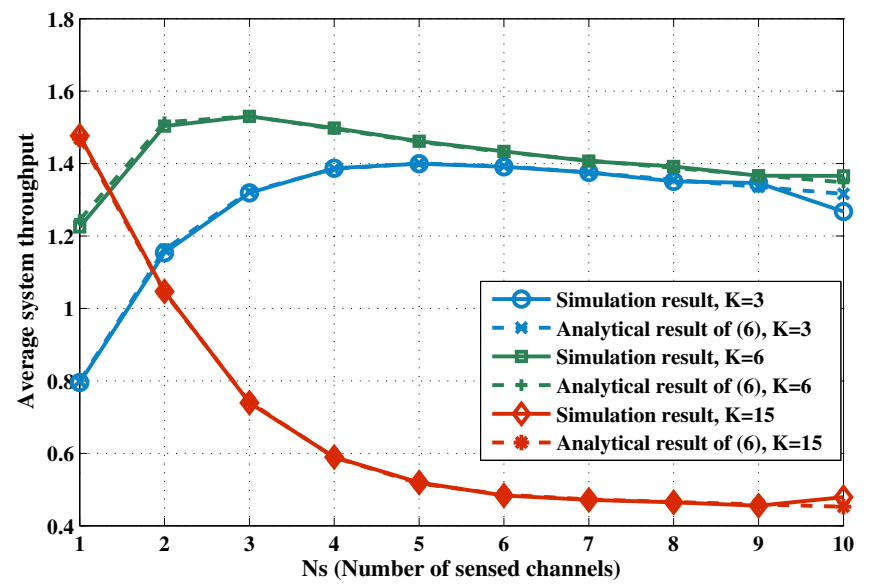

Fig. 6. Average system throughput when $M=4$ and $p_{\text {tra }}=0.8$.

SUs for each $M$ value. As depicted in Fig. 7, the optimal number of $S U \mathrm{~s}$ is equal or close to $M$.

Fig. 8 and Fig. 9 illustrate the system throughput under fixed $M$ and random $\mathrm{M}$ with increasing values of $N_{s}$, respectively. The analytical results under a random $\mathbf{M}$ match closely with the simulation results. It is observed that the system throughput under a fixed $M$ and a random $\mathbf{M}$ follow the same trend when 


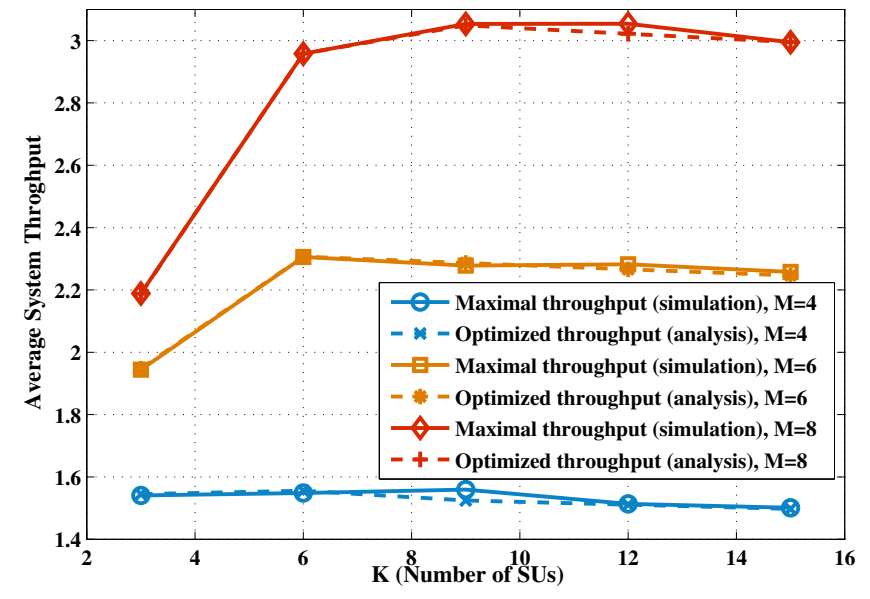

Fig. 7. Optimal average system throughput.

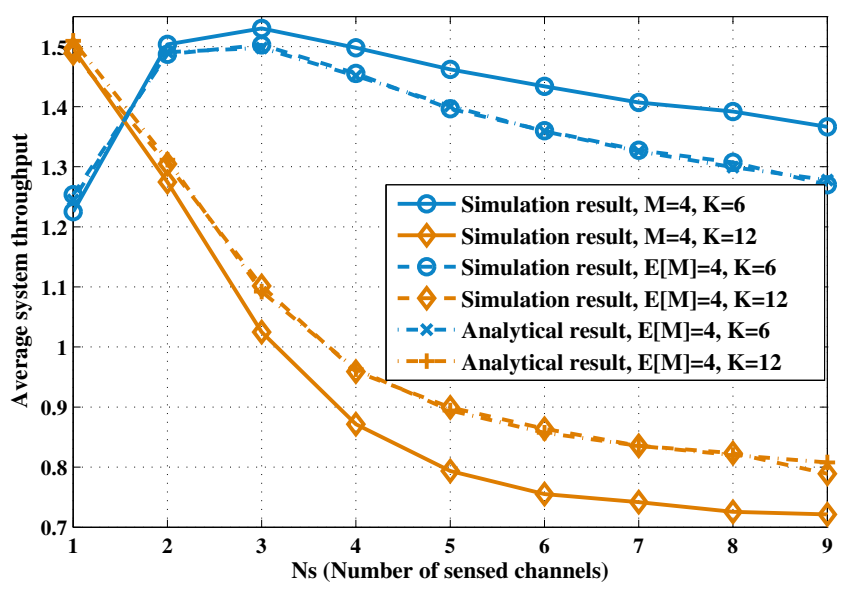

Fig. 8. Average system throughput when $E[\mathbf{M}]=4$.

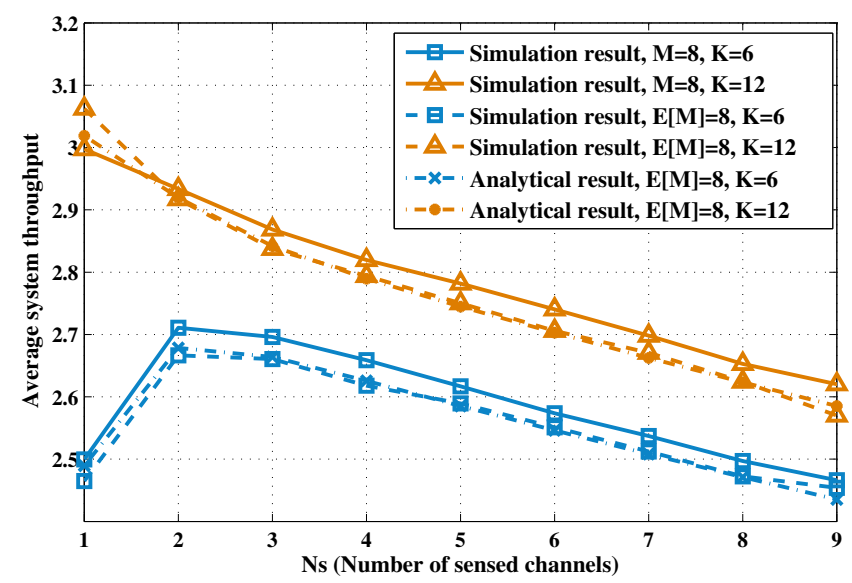

Fig. 9. Average system throughput when $E[\mathbf{M}]=8$.

$E[\mathbf{M}]=q N=M$. That means Theorem 3 can be also applied to the situations with random number of idle channels based on $E[\mathbf{M}]$. Specifically, the system throughputs under a fixed $M$ and a random $\mathbf{M}$ attain the same value when $N_{s}=1$. In addition, the system throughput curve under a random $\mathbf{M}$ is parallel with that under a fixed $M$ when $N_{s} \geq N-M+1$.

In Fig. 10, the average system throughput under imperfect sensing is plotted as a function of the distance $D_{s u}$. It is

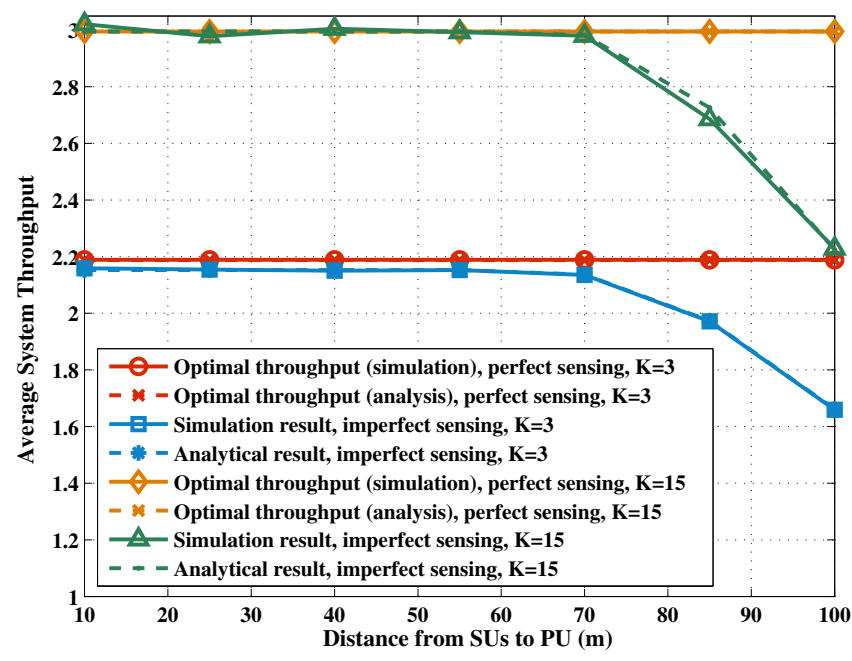

Fig. 10. Average system throughput under perfect and imperfect sensing.

TABLE I

OPTIMAL $\left(N_{s}, p_{\text {TRA }}\right)$ FOR THROUGHPUT MAXIMIZATION

\begin{tabular}{cccccc}
\hline & $K=3$ & $K=6$ & $K=9$ & $K=12$ & $K=15$ \\
\hline$M=4$ & $(5,1)$ & $(2,1)$ & $(1,1)$ & $(1,0.83)$ & $(1,0.67)$ \\
\hline$M=6$ & $(4,1)$ & $(2,1)$ & $(1,1)$ & $(1,0.83)$ & $(1,0.67)$ \\
\hline$M=8$ & $(3,1)$ & $(2,1)$ & $(1,1)$ & $(1,0.83)$ & $(1,0.67)$ \\
\hline
\end{tabular}

noted that the growing $D_{s u}$ will reduce the SNR, $\gamma$, at the $\mathrm{SU}$ receiver, which in turn leads to an increasing $P_{f}$ according to (20). The results show the effects of the physical fading channel on the SU spectrum sensing performance. At the optimal point $\left(N_{s}^{*}, p_{\text {tra }}^{*}\right)$ attained based on Theorem 3 , the system throughput under perfect sensing is constant under different $D_{s u}$, while the system throughput under imperfect sensing reduces as $D_{s u}$ grows. That implies it is also beneficial to reduce the false alarm probability $P_{f}$ to maximize the system throughput.

\section{CONCLUSION}

In this paper, we have introduced the "extended sensing strategy" in which each SU randomly selects a subset of channels out of a spectrum of $N$ channels for sensing. Following that, each SU transmits the packet in any one of the detected idle channels. The slotted ALOHA scheme is utilized for access to idle channels. From the analysis, we conclude that the effective number of sensing channels $N_{s}$ is dependent on the total number of licensed channels $N$ and the number of the idle channels $M$, i.e., $N_{s} \leq N-M+1$. The system throughput under a random number of idle channel $\mathbf{M}$ is also analyzed and compared with that under a fixed number of idle channels $M$. Then, the optimal $N_{s}$ is analyzed and reveals the relationship between $N_{s}$ and the average system throughput. Later on, we formulated a joint optimization problem with respect to $\left(N_{s}, p_{\text {tra }}\right)$ and obtained the optimal system throughput. Thus, notice that sensing strategy is related to the packet transmission probability. That means to improve the system throughput, each SU should take both $N_{s}$ and $p_{\text {tra }}$ in consideration. Finally, we have analyzed the average system throughput under the imperfect sensing. Comparing the simulation and analytical results, it is concluded that 
the system throughput from our analysis almost arrives at the simulation results. Further, besides the slotted ALOHA scheme, we can also apply the "extended sensing strategy" to other effective MAC schemes, which will be one of our future researches.

\section{APPENDIX}

Let $X_{i j}$ be the indicator random variable, where $i \in$ $\{1,2, \ldots, M\}, j \in\{1,2, \ldots, U\}$, and $U$ is the total number of packets to be transmitted from all SUs within one $T_{P m}$. So that $X_{i j}=1$ if $j^{\text {th }}$ the packet selects the $i^{\text {th }}$ idle channel for access, otherwise $X_{i j}=0$, and $X_{i}=\sum_{j=1}^{K} X_{i j}$ indicates the number of packets which select the $i^{\text {th }}$ idle channel. For each $X_{i j}$ represents a Bernoulli trial, the probability that $j^{\text {th }}$ packet (or any one packet) selects the $i^{\text {th }}$ idle channel (any given channel) for access when $M_{s}$ idle channels are detected, can be presented as,

$$
\begin{aligned}
p_{s a c \mid M_{s}}^{(i)} & =p_{i d l}\left(M_{s}\right) \cdot\left(\begin{array}{c}
M-1 \\
M_{s}-1
\end{array}\right) /\left(\begin{array}{c}
M \\
M_{s}
\end{array}\right) \cdot \frac{1}{M_{s}} \\
& =p_{i d l}\left(M_{s}\right) \cdot \frac{1}{M}
\end{aligned}
$$

where

$$
p_{i d l}\left(M_{s}\right)=\left(\begin{array}{c}
M \\
M_{s}
\end{array}\right)\left(\begin{array}{c}
N-M \\
N_{s}-M_{s}
\end{array}\right) /\left(\begin{array}{c}
N \\
N_{s}
\end{array}\right)
$$

is the probability of $M_{s}$ idle channels sensed by one SU and the second term in (28a) is the probability of $i^{\text {th }}$ idle channel included in the $M_{s}$ idle channels. The probability of the packet selecting the $i^{\text {th }}$ idle channel out of the $M_{s}$ idle channels is $1 / M_{s}$ as presented in the third term in (28a), since the probability of selecting any one idle channel within $M_{s}$ idle channels is equal to each other. Hence, the probability of the $j^{\text {th }}$ packet selecting the $i^{\text {th }}$ channel, $p_{s a c}^{(i)}$, is

$$
\begin{aligned}
p_{s a c}^{(i)} & =p\left(X_{i j}=1\right)=\sum_{M_{s}=1}^{\min \left\{M, N_{s}\right\}} p_{s a c \mid M_{s}}^{(i)} \\
& =\frac{1}{M} \sum_{M_{s}=1}^{\min \left\{M, N_{s}\right\}} p_{i d l}\left(M_{s}\right)=\frac{1}{M} p_{i d l}\left(M_{s} \geq 1\right) \\
& =\frac{1}{M}\left(1-p_{i d l}\left(M_{s}=0\right)\right),
\end{aligned}
$$

Thus, if $N_{s} \geq N-M+1$, it will always contains at least one idle channel within the $N_{s}$ detected channels which means $p_{i d l}\left(M_{s}=0\right)=0$. While, if $N_{s} \leq N-M$, the packet will fail to access when the channels included in $N_{s}$ sensed channel are all occupied by PUs, which implies no idle channel within $N_{s}$ channels, so

$$
\begin{aligned}
p_{\text {idl }}\left(M_{s}=0\right) & = \begin{cases}\left(\begin{array}{c}
N-M \\
N_{s}
\end{array}\right) /\left(\begin{array}{c}
N \\
N_{s}
\end{array}\right), & \text { if } N_{s} \leq N-M ; \\
0, & \text { if } N_{s} \geq N-M+1 ;\end{cases} \\
& = \begin{cases}\frac{\left(N-N_{s}\right]^{[M]}}{N^{[M]},} & \text { if } N_{s} \leq N-M ; \\
0, & \text { if } N_{s} \geq N-M+1 ;,\end{cases}
\end{aligned}
$$

where $L^{[k]}=L(L-1) \cdots(L-(k-1))$. Consequently, the probability, $p_{s a c}^{(i)}$, of one packet selecting the $i^{\text {th }}$ idle channel accessing is

$$
\begin{aligned}
p_{\text {sac }}^{(i)} & =\frac{1-p_{\text {idl }}\left(M_{s}=0\right)}{M} \\
& = \begin{cases}\frac{1}{M}\left(1-\frac{\left(N-N_{s}\right]^{[M]}}{N^{[M]}}\right), & \text { if } N-M \geq N_{s}, \\
\frac{1}{M}, & \text { else. }\end{cases}
\end{aligned}
$$

From (32), it can be observed that $p_{s a c}^{(i)}$ is not related to $M_{s}$. That implies that accessing a given idle channel will not be affected by the number of the idle channels sensed by an SU.

To prove the monotonic property of $p_{s a c}^{(i)}$, we first relax $N_{s}$ as $0<\bar{N}_{s} \leq N-M$. Then we can differentiate $p_{s a c}^{(i)}$ as followings,

$$
\begin{aligned}
\frac{\partial p_{s a c}^{(i)}}{\partial \bar{N}_{s}} & =\Upsilon \sum_{j=0}^{M-1} \frac{1}{\left(N-\bar{N}_{s}-j\right)}>0, \\
\frac{\partial^{2} p_{s a c}^{(i)}}{\partial \bar{N}_{s}^{2}} & =\Upsilon \sum_{j=0}^{M-1} \frac{1}{\left(N-\bar{N}_{s}-j\right)^{2}}-\frac{\left(\frac{\partial p_{s a c}^{(i)}}{\partial N_{s}}\right)^{2}}{\Upsilon}<0,
\end{aligned}
$$

where $\Upsilon=\frac{\left(N-\bar{N}_{s}\right]^{[M]}}{M \cdot N^{[M]}}$. Through differentiating (32) with respect $\bar{N}_{s}$, we can know the probability $p_{\text {sac }}$ is an increasing function versus $N_{s}$.

\section{REFERENCES}

[1] J. M. III, "Cognitive radio: an integrated agent architecture for software defined radio," Ph.D. thesis, KTH (Royal Institute of Technology), 2000.

[2] C. Cordeiro, K. Challapali, and M. Ghosh, "Cognitive PHY and MAC layers for dynamic spectrum access and sharing of TV bands," in Proc. 2006 ACM International Workshop on Technology and Policy for Accessing Spectrum, p. 3.

[3] B. Hamdaoui and K. Shin, "OS-MAC: an efficient MAC protocol for spectrum-agile wireless networks," IEEE Trans. Mobile Comput., vol. 7, no. 8, pp. 915-930, 2008.

[4] H. Su and X. Zhang, "Cross-layer based opportunistic MAC protocols for QoS provisionings over cognitive radio wireless networks," IEEE J. Sel. Areas Commun., vol. 26, no. 1, pp. 118-129, 2008.

[5] — "Design and analysis of a multi-channel cognitive MAC protocol for dynamic access spectrum networks," in Proc. 2008 IEEE Military Communications Conference, pp. 1-7.

[6] S. Choe and S.-K. Park, "Throughput of slotted aloha based cognitive radio MAC," in Proc. 2009 International Conference on Ubiquitous Information Technologies Applications, pp. 1-4.

[7] S. Choe, "Performance analysis of slotted aloha based multi-channel cognitive packet radio network," in Proc. 2009 IEEE Consumer Communications and Networking Conference, pp. 1-5.

[8] R. Rajbanshi, "OFDM-based cognitive radio for DSA networks," Information and Telecommunication Technology Center, The University of Kansas, tech. rep., 2007.

[9] I. F. Akyildiz, W.-Y. Lee, M. C. Vuran, and S. Mohanty, "Next generation/dynamic spectrum access/cognitive radio wireless networks: a survey," Computer Networks, vol. 50, no. 13, pp. 2127-2159, 2006.

[10] Y.-C. Liang, Y. Zeng, E. Peh, and A. T. Hoang, "Sensing-throughput tradeoff for cognitive radio networks," IEEE Trans. Wireless Commun., vol. 7, no. 4, pp. 1326-1337, 2008.

[11] Z. Quan, S. Cui, A. Sayed, and H. Poor, "Optimal multiband joint detection for spectrum sensing in cognitive radio networks," IEEE Trans. Signal Process., vol. 57, no. 3, pp. 1128-1140, 2009.

[12] L. Luo, C. Ghosh, and S. Roy, "Joint optimization of spectrum sensing for cognitive radio networks," in Proc. 2010 IEEE Global Telecommunications Conference, pp. 1-5.

[13] L. Luo, N. Neihart, S. Roy, and D. Allstot, "A two-stage sensing technique for dynamic spectrum access," IEEE Trans. Wireless Commun., vol. 8, no. 6, pp. 3028-3037, June 2009. 
[14] X. Li, H. Liu, J. Zhang, and P. Zhang, "A novel method for system parameters estimation in distributed cognitive radio networks," submitted to IEEE Commun. Lett., 2012.

[15] Q. Chen, M. Motani, W.-C. Wong, and Y.-C. Liang, "Opportunistic spectrum access protocol for cognitive radio networks," in Proc. 2011 IEEE International Conference on Communications, pp. 1-6.

[16] T. Rappaport, et al., Wireless Communications: Principles and Practice, Vol. 207. Prentice Hall PTR, 1996.

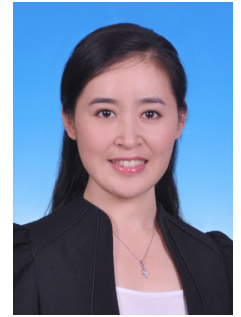

Xiaofan Li received the B.S. degrees from Beijing University of Posts and Telecommunications (BUPT), Beijing, China. She is currently working towards her Ph.D. degree at Key Lab of Universal Wireless Communications, Ministry of Education in BUPT. From September 2010 to September 2011, she studied in University of Washington as an exchanged Ph.D. student. She was awarded "Outstanding Graduate Student" of BUPT. Her current research interests include resource management in cooperative communication and throughput analysis in distributed cognitive radio system.

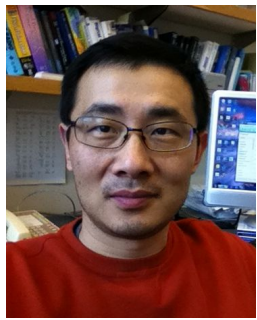

Hui Liu , ZhiYuan Chair Professor, received his B.S in 1988 from Fudan University, Shanghai, China, and a Ph.D. degree in 1995 from the Univ. of Texas at Austin, all in electrical engineering. He was previously an assistant professor at the Dept. of EE at Univ. of Virginia and a full professor at the Dept. of EE, Univ. of Washington. Dr. Liu was one of the principal designers of the TD-SCDMA technologies. $\mathrm{He}$ founded Adaptix in 2000 and pioneered the development of OFDMA-based mobile broadband networks (mobile WiMAX and 3G LTE). Dr. Liu is the creator of CMMB transmission technology which enables the delivery of mobile TV services to more than a billion of population in different regions of the world.

Dr. Liu has published 45 journal articles and has 67 awarded patents. He is the author of OFDM-Based Broadband Wireless Networks: Design and Optimization (Wiley, 2005) and Signal Processing Applications in CDMA Communications (Artech House, 2000). He was selected a Fellow of IEEE for contributions to global standards for broadband cellular and mobile broadcasting. He is the General Chairman for the 2005 Asilomar conference on Signals, Systems, and Computers. He is a recipient of 1997 National Science Foundation (NSF) CAREER Award, the Gold Prize Patent Award in China, and 2000 Office of Naval Research (ONR) Young Investigator Award.

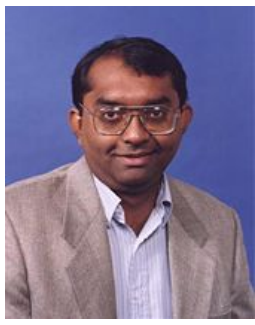

Sumit Roy received the B. Tech. degree from the Indian Institute of Technology (Kanpur) in 1983, and the M. S. and Ph. D. degrees from the University of California (Santa Barbara), all in Electrical Engineering in 1985 and 1988 respectively, as well as an M. A. in Statistics and Applied Probability in 1988. Presently he is Professor of Electrical Engineering, Univ. of Washington where his research interests include analysis/design of wireless communication and sensor network systems with a diverse emphasis on various technologies: wireless LANs (802.11) and emerging 4G standards, multi-standard wireless inter-networking and cognitive radio platforms, vehicular and underwater networks, and sensor networking involving RFID technology.

He spent 2001-03 on academic leave at Intel Wireless Technology Lab as a Senior Researcher engaged in systems architecture and standards development for ultra-wideband systems (Wireless PANs) and next generation high-speed wireless LANs. During Jan-July 2008, he was Science Foundation of Ireland's E.T.S. Walton Awardee for a sabbatical at University College, Dublin and was the recipient of a Royal Acad. Engineering (UK) Distinguished Visiting Fellowship during summer 2011. His activities for the IEEE Communications Society (ComSoc) includes membership of several technical and conference program committees, notably the Technical Committee on Cognitive Networks. He currently serves on the Editorial Board for IEEE Trans. Communications and IEEE Intelligent Transportation Systems. He was elevated to IEEE Fellow by Communications Society in 2007 for his "contributions to multiuser communications theory and cross-layer design of wireless networking standards."

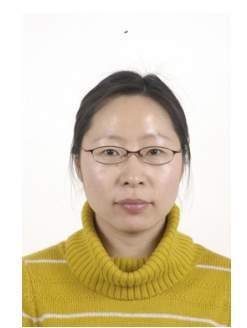

Jianhua Zhang received her Ph.D. degree in circuit and system from Beijing University of Posts and Telecommunication (BUPT) in 2003. She is an associate professor from 2005 and now she is professor of BUPT. She has published more than 100 articles in referred journals and conferences. She was awarded "2008 Best Paper" of the Journal of Communication and Network. In 2009, she received second prize award by CCSA for her contributions to ITU-R and 3GPP in IMT-Advanced channel model. In 2011, she was awarded the "New Century Excellent Talents in University" by MOE and "Young Talent Teachers" by Fok Ying Tung Education Foundation. Her current research interests include propagation models, green techniques for IMT-Advanced and beyond system, Relay, CoMP and Cognitive Radio techniques, etc.

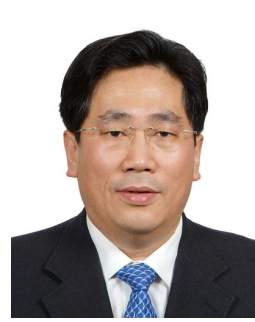

Ping Zhang Ph.D. and Prof. of BUPT, focuses research on wireless communication, new technologies for cognitive radio, cognitive wireless networks, TD-LTE, MIMO, OFDM and etc. He is Executive Associate Editor-in-chief on information sciences of Chinese Science Bulletin, a Member of nextgeneration broadband wireless communication network in National Science and Technology Major Project committee, a Member of the 5th Advisory Committee of NSFC(National Natural Science Foundation of China), the Chief Scientist of "973" National Basic Research Program of China, He applied over 114 patents with 51 authorized patents. He received award of National Science and Technology Advance Prize twice, Award of National Science and Technology Invention Prize once, the Provincial Science and Technology Awards many times,and was given the Title of Outstanding Scientific and Technological Workers in 2010.

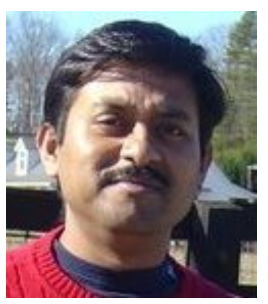

Chittabrata Ghosh is currently a Senior Researcher at Nokia Research Center (NRC), Berkeley, CA since July 2011. Before joining NRC, he was a Postdoctoral Researcher at University of Washington, Seattle since July 2009. He had received Ph.D. degree from Department of Computer Science, University of Cincinnati, OH in 2009. He is actively involved in IEEE standardization activities focusing on system design and architecture for low power WiFi networks. Apart from this, his research interests include analysis of coexistence issues among various cooperating wireless networks and MAC layer optimization techniques for wireless sensor networks. He is also leading Nokia's university research collaborations on multi-faceted research and policy issues in the TV White Spaces. His activities for the IEEE Communications Society (ComSoc) include membership of several technical and conference program committees. $\mathrm{He}$ is also the Vice Chair and Co-Editor of the Technical Committee on Simulations under the IEEE Computer Society. 